\title{
Full Field Nanoimprint on Mask Aligners Using Substrate Conformal Imprint Lithography Technique*
}

\author{
Ran Ji, A. KrÜGer, M. Hornung \\ SUSS MicroTec Lithography GmbH, Garching, Germany \\ M. Verschuuren, R. VAn de LaAR and J. van Eekelen \\ Philips Research, Eindhoven, Netherlands
}

\section{Introduction}

Nowadays, the development of integrated circuit (IC) industry and scientific researches rely more and more on the nanofabrication technologies. The resolution limits of optical lithography are very real even with a number of "optical tricks" [1] at work. E-beam lithography (EBL) provides excellent resolution down to several nanometers. However, due to its scanning exposure principle the throughput of EBL is too low for mass production or even for research that requires multiple identical samples e.g. (bio)sensors. Nanoimprint lithography (NIL) [2] has been included on the International Technology Roadmap for Semiconductors (ITRS) lithography roadmap [3] for $32 \mathrm{~nm}, 22 \mathrm{~nm}$ and $16 \mathrm{~nm}$ nodes. This parallel patterning technique shows great potentials in fabrication of nanostructures with high resolution at low costs.

Among the potential applications of NIL techniques, global light emitting (LED) diode market grew rapidly in the last years. Photonic crystal $(\mathrm{PhC})$ structures in LEDs have been demonstrated to improve the output efficiency by diffracting waveguide modes. As yet, $\mathrm{PhC}$ LEDs have not been introduced as standard lighting because the process of fabricating photonic structures by advanced lithographic techniques is time consuming and costly. NIL techniques have attracted considerable attention in this area due to its high resolution and low cost of ownership (CoO). There are several NIL candidates in the market: hot embossing, UV-NIL [4] stepper with hard stamps and NIL with soft stamps. Hot embossing has been disqualified out from this game due to its thermal cycles. The throughput of the current NIL steppers in the market is still too low to meet the requirement of mass production and it should be increased by factor 4 to compete with the mainstream $193 \mathrm{~nm}$ immersion (193i) lithography. In addition, since most of the LED fabrication atmospheres are above class 10000 ,

\footnotetext{
* contact: Andreas Krueger, SUSS MicroTec Lithography GmbH, Schleissheimer Str. 90, 85748 Garching, Germany email: andreas.krueger@suss.com www.suss.com
}

this technique is limited in a transit stage to production due to the strictly particle sensitive rigid (quartz) stamp and process. Soft imprint techniques [5] allow for large area imprinting and are less sensitive to particles. However, the resolution is usually limited to several hundreds nanometers due to stamp distortion caused by pressure. A novel imprint technique developed by Philips Research and SUSS MicroTec, substrate conformal imprint lithography (SCIL [6]) bridges the gap between small rigid imprint stamp for best resolution and large-area soft imprint with limited resolution. Based on a cost-effective upgrade on SUSS mask aligner, the capability can be enhanced to nanoimprint of sub-10 nm structures on up to 6 inch area without affecting any established conventional optical lithography processes on the machine.

In order to reduce the $\mathrm{CoO}$ of large-area imprint stamp, SCIL process uses flexible multilayer working stamps [7] replicated from the original master pattern. Before stamp replication, the silicon master surface needs to be modified with $1 \mathrm{H}, 1 \mathrm{H}, 2 \mathrm{H}, 2 \mathrm{H}-$ -perfluorodecyltrichlorosilane chemistry as anti-adhesive layer [8] in order to prevent risk of adhesion of the polydime-thylsiloxane (PDMS) stamp material. The PDMS working stamp is replicated from the master and simultaneously glued onto a thin glass carrier. The composite stamp combines the advantages of in-plane stiffness of glass carrier and the softness of PDMS stamp. To achieve a wafer scale conformal contact between working stamp and substrate, the SCIL process relies on a sequential imprinting process. In addition, capillary forces help pulling the stamp into the liquid imprint resist. The approaching of the flexible stamp starts from one side and spreads to the whole stamp subsequently by releasing the vacuum in the grooves step by step and applying a small overpressure of 20 mbar on the stamp. This sequent contact mechanism prevents the flexible stamp from trapping air and therefore ensures that the stamp follows exactly the undulating topography over whole substrate surface. The imprint resists that are used are wetting on the PDMS and the resulting capillary forces fill up features with resist. The capillary forces are leading 
which minimizes the structure deformation and lateral stamp distortion during the imprint process. In this way sub-50 nm resolution patterns have already been demonstrated.

\section{Experimental and results}

In this paper, a 6 inch silicon master (Fig. 1) from AMO GmbH (Aachen, Germany) with 2D holes array fabricated by laser interference lithography and etching processes was replicated by SCIL. Full field imprint with the replicated SCIL stamp has been carried out into a $120 \mathrm{~nm}$ thick imprint sol-gel layer on a 6 inch silicon wafer substrate. After removal of the residual layer (ca. $20 \mathrm{~nm}$ ) by reactive ion etching (RIE), the structures have been transferred into silicon substrate to a depth of $300 \mathrm{~nm}$. Finally, the imprint sol-gel layer has been stripped by HF dipping. Figure 2 shows the imprinted wafer after structure transfer and resist stripping. This demonstrates the imprint uniformity and structure fidelity of SCIL process.

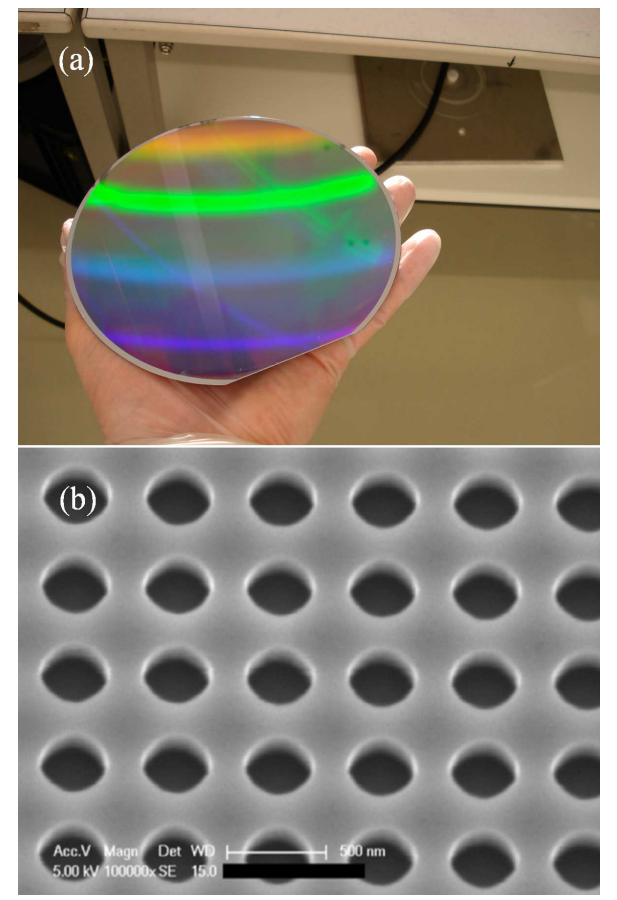

Fig. 1. (a) Photograph and (b) SEM image of the silicon master with $2 \mathrm{D}$ holes array made by laser interference lithography and etching processes; the pitch is $513 \mathrm{~nm}( \pm 5 \%$ over 6 inch area); the diameter and the depth of the holes are $340 \mathrm{~nm}$ and $200 \mathrm{~nm}$, respectively.

In addition to sol-gel process, we have also demonstrated an UV enhanced SCIL process where we used AMONIL (AMO GmbH, Germany) resist as imprint resist for SCIL process. Figure 3 shows scanning electron microscopy (SEM) images of various imprinted structures made with the UV-SCIL process. The benefit from the UV-curing is that the process time can be optimized to

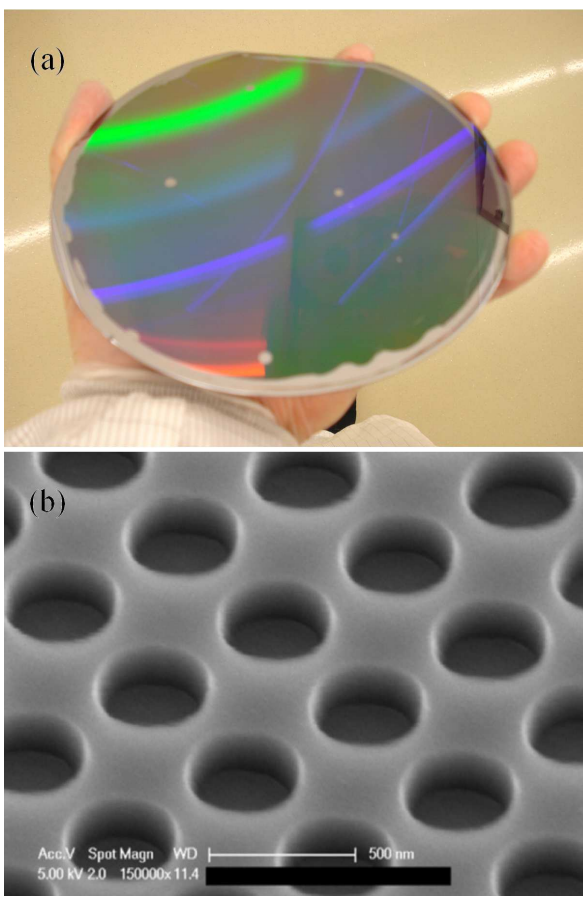

Fig. 2. (a) Photograph and (b) SEM image of the imprinted wafer after structure transfer and stripping of the imprint sol-gel.
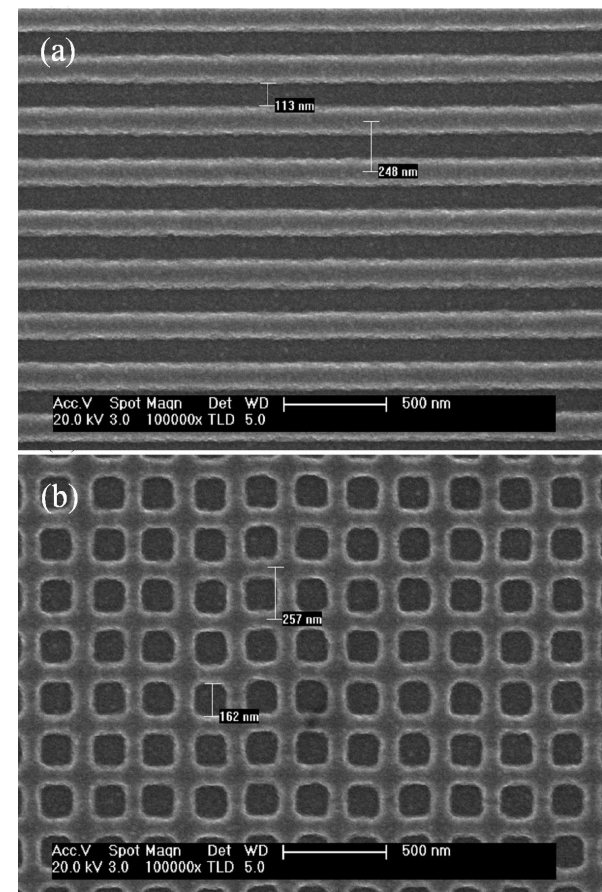

Fig. 3. SEM images of UV-SCIL results: (a) lines and spaces structures with a pitch of $248 \mathrm{~nm}$ and a line width of ca. $135 \mathrm{~nm}$; (b) imprinted square shape structures array, the side length is $162 \mathrm{~nm}$ and the space between two squares is $c a .100 \mathrm{~nm}$. 
less than 2 min per wafer which increases the throughput dramatically in comparison to sol-gel imprint resist, which requires around $10 \mathrm{~min}$.

In summary, a revolutionary novel NIL technique and the corresponding tooling solution on SUSS mask aligners, SCIL, have been introduced. The SCIL process allows large area imprinting on 6 inch wafers with resolution down to sub-10 nm. This technique shows therefore great potential in high volume production due to its excellent reliability, especially for patterned media storage and high brightness LED applications.

\section{References}

[1] A.C. Tobey, Electronics 52, 109 (1979).

[2] S.Y. Chou, P.R. Krauss, P.J. Renstrom, J. Appl. Phys. Lett. 67, 3114 (1996).
[3] International technology roadmap for semiconductors, 2003 edition, "lithography", http:// www.itrs.net/links/2003ITRS/Litho2003.pdf .

[4] M. Bender, M. Otto, B. Hadam, B. Vratzov, B. Spangenberg, H. Kurz, Microelectron. Eng. 53, 233 (2000).

[5] M. Bender, U. Plachetka, J. Ran, A. Fuchs, B. Vratzov, H. Kurz, T. Glinsner, F. Lindner, J. Vac. Sci. Technol. B 22, 3229 (2004).

[6] M. Verschuuren, H. van Sprang, Mater. Res. Soc. Symp. Proc. 1002, 1002-N03-05 (2007).

[7] U. Plachetka, M. Bender, A. Fuchs, T. Wahlbrink, T. Glinsner, H. Kurz, Microelectron. Eng. 83, 944 (2006).

[8] M. Beck, M. Graczyk, I. Maximov, E.-L. Sarwe, T.G.I. Ling, M. Keil, L. Montelius, Microelectron. Eng. 61-62, 441 (2001). 